\title{
Elemental imaging and speciation in plant science
}

\author{
Jörg Feldmann • Eva M. Krupp
}

Published online: 14 February 2012

(C) Springer-Verlag 2012

Plants are fascinating and complex organisms which are generally immobile and utilise the resources of their immediate environment to thrive.

As a matter of fact, plants are at the base of the food chain, and represent the main source of food for higher terrestrial organisms, including human beings; for example, rice is a staple food for entire populations in Asia. The nutritive value of a plant itself is complemented by a plethora of trace elements that are present in its fruit and other edible plant parts; indeed, this is the main source of essential trace elements for the human population. However, plants can also take up and store nonessential elements, including those toxic or hazardous to our health. The extent to which plants take up trace elements depends largely on the environment in which the plant is growing, but not every plant reacts in the same way to the trace elements in its environment: different plants have different but specific nutritive requirements, and are more or less tolerant of toxic elements.

In a world facing serious problems regarding how to feed the ever-growing population on the basis of dwindling

Published in the special paper collection Elemental Imaging and Speciation in Plant Science with guest editors J. Feldmann and E. Krupp.

\section{J. Feldmann $(\bowtie)$}

Environmental Analytical Chemistry, Trace Element Speciation Laboratory (TESLA), College of Physical Sciences,

University of Aberdeen,

Aberdeen AB24 3UE, Scotland, UK

e-mail: j.feldmann@abdn.ac.uk

\section{E. M. Krupp}

Environmental Analytical Chemistry, Aberdeen Centre for Environmental Sustainability (ACES), College of Physical Sciences, University of Aberdeen,

Aberdeen AB24 3UE, Scotland, UK arable land, with vast areas polluted through mining or other anthropogenic activities, it is imperative to understand how plants take up the essential elements that we rely on for a healthy living, and the mechanisms they use to cope with pollution through toxic elements.

During their evolution, plants have developed strategies to acquire nutrients and essential elements in the amounts or concentrations that they require, while toxic elements may be excluded or detoxified in plants. These strategies are based on the biochemical machinery of the plant's various cells, in the form of transporter molecules or chelators such as siderophores or phytochelatins, which enable elemental homeostasis to be achieved in a variety of different habitats. The uptake, (short- and long-range) transport and translocation of an element in a plant depend mostly on the distinct molecular occurrence of the element in the soil and the cell compartment, the actual element itself, as well as the organism and its genetic predisposition. Hence, in order to predict the distribution of elements in a plant, we need to know the molecular structures of the species of these element-their speciation-at the soil/plant interface as well as inside the different cells and cell compartments at all times.

In order to understand the underlying mechanisms, it is useful to determine all of the molecular structures of all of the elements of interest in all cell compartments. Since this concept has some similarities to the proteomics approach, it is now widely termed "metallomics." Although the current approach does not necessarily satisfy the condition that all of the species of all of the elements of interest (or one element) must be identified and quantified in a certain biological substructure, the approach in which the most important biologically active species of an element is determined may enable molecular biologists to link these species to proteins and to the encoding genes. 
Different analytical approaches have been proposed recently that make it possible to determine the elements linked to different plant parts with individual sensitivity as well as lateral resolution. Sophisticated methodologies to quantify selected elemental species or the entire metallome have been developed simultaneously. Therefore, we believe that it is important and timely to alert the plant science community to these developments using this special issue of Analytical and Bioanalytical Chemistry, in which a series of critical reviews and original papers about elemental speciation studies and the elemental imaging of plants are covered.

This special issue contains five major reviews, which are complemented by original papers and a technical note.

The reviews cover elemental imaging that provides insight into the spatial distributions of elements in different plant parts using a variety of state-of-the-art analytical techniques, such as synchrotron radiation, nanoSIMS, or laser ablation ICPMS, in order to localise trace elements with high spatial resolution, as well as molecular and elemental speciation studies using multiple analytical techniques.

Multi-technique approaches are usually necessary to solve complex analytical problems such as determining unstable trace element species in a biological sample. The original contributions also describe the use a variety of different analytical techniques, ranging from NMR fingerprinting in tree bark to multi-dimensional chromatography with dual elemental and molecular mass spectrometric detection (such as ICP-MS and ESI-MS) for the determination of arsenic species in seaweed, or the identification and possibly quantification of metallopeptides and metalloproteins.

Overall, we believe that the collection of papers assembled in this issue of Analytical and Bioanalytical Chemistry provides valuable information for interested researchers by highlighting new analytical methods, critically evaluating limitations, and discussing challenges that the analytical community may face in the coming years.

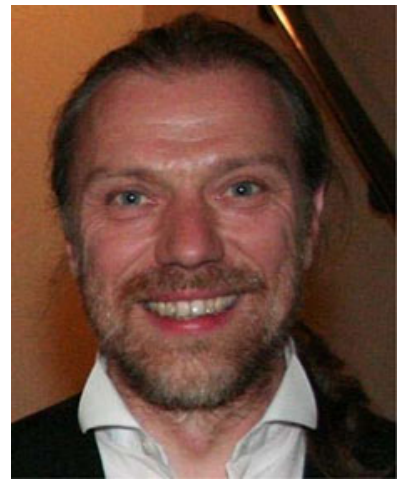

Jörg Feldmann

is a professor at the University of Aberdeen and has held the Chair in Environmental Analytical Chemistry there since 2004. He obtained his Ph.D. in 1995 from the University of Essen (Germany), and was a Feodor Lynen Fellow (Alexander von Humboldt) at the University of British Columbia in Vancouver (Canada) before moving to Scotland in 1997 to become a lecturer at the University of Aberdeen. He is currently leading TESLA (Trace Element Speciation Laboratory Aberdeen) as part of the newly founded Marine Biodiscovery Centre. He is a member of the Advisory Boards for Environmental Chemistry and Analytical and Bioanalytical Chemistry, and has published more than 150 scientific papers-mainly on element speciation analysis in environmental and biological systems - and given more than 100 invited and plenary lectures at international conferences. The main focus of his research is on elemental speciation, with an emphasis on the development of hyphenated mass spectrometry for studies of the molecular forms of arsenic and their behaviour in biology and the environment.

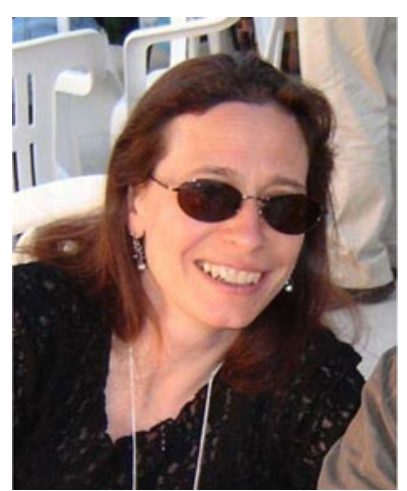

Eva Maria Krupp obtained her Ph.D. in analytical chemistry at the University of Essen, Germany, in 1999. She then moved to the CNRS for Bioinorganic Chemistry at the University of Pau, France, to work as a postdoctoral fellow in the element speciation group of O.F.X. Donard. From there she moved to the University of Aberdeen, Scotland, in 2005, and was appointed a lecturer in analytical and environmental chemistry in 2008 within TESLA (Trace Element Speciation Laboratory Aberdeen) and the Aberdeen Centre for Environmental Sustainability (ACES), University of Aberdeen. She is a committee member of the Royal Society of Chemistry's Analytical Division (Scotland). Her research is focussed on element speciation and related method developments, and currently on mercury in the environment and life in particular. She has published 45 scientific papers and given more than 20 invited lectures and workshops at international conferences and symposia. 